\title{
Intento de suicidio en niños: algunos aspectos biodemográficos
}

\author{
Mario Valdivia P.'; Claudia Schaub M.²; Marcela Díaz P.²
}

\begin{abstract}
Resumen
Objeisvo: describis algunas coracteristicas bıodemográficas y de salud mental er. niños que intentaron suicidia, asi cono lcs circunisancias del inento. Pacientes y mélodo: se revisoron retrospectivamente los antecedentes clinicos de trenra cascs de intentos de suicidic hospitalizados en el servicio de pediatría del Hospital Regionol de Concepción. entre octubre de 1995 y mayo de 1997. En la mayoría de los cosos se habia hecho una evaluación psiquiárica durante lo hospitalizacion. Resuricodos: lo edac de los ofectodos fluctuaba entre 7 y 15 años. La mavoría eran mujeres $\mid 90 \%$. sj́c $36,7 \%$ vivían con ombos padres v cerco de $75 \%$ tenion algún trosiono psiquiálrico iprinctipo|mente depresión El mílodo más comúnmente utilizado en el intento fue la ingestión de medicarnentos. En la mayor pare de los casos los intentos obedecieron a acros impulsivos desencadenados por conflicros fami iares. Sólo $23.3 \%$ de los pacientes continuaba en Iratamiento psiquićtrico uno a veinte meses después de egresar del hospital. Conclusićn: el conocimiento adec para e-fenrar y resolver correctamente el problema de estos nin̆os.
\end{abstract}

ipaiabras clave: suicidio, infento, depresión.

\section{Suicidal attempts in children: some biodemographic aspects}

Objetwe to describe some biodemographic ospects of children who were admited to a general hospilal ater attemped suicide Forients ano' methods: clinical recoras of all thirty coses of allempled suicide thal were hospilatized $n$ the ped atric wads of a universivy associaled pubiic general hospital at Concepción. Chile, between october 1995 and mcy 1907 were reviewed for intormation on demographic aspects cnd those related wilh the suicidal alterpt. Results polient age varied from 7 years to i 5 yeors. They were mosly Female $180 \%$, and only $36.7 \%$ lived with both parents. Nearly 75\% of re ctildren and odolescents hoc a psychiotric disease [mosily a depressive disorderi. The most commonly employed method for the suicidal altempt was crug ingestion. In mosl cases suicidal attempt was the resiff of an impulsive action after c family conflict Only $23.3 \%$ of the salienls were still under psychialric treatmen or supervision one to twenty months ofter hespital discharge. Conclusion: more knowledge and better undestanding of attempted sucide in ch laten is verv imporant for pediot cians becouse they are usuaily the first heaith professionals to toke core ct these cases

ikey words: surcide, altempled. depressive disorder.)

Las conductas suicidas en niños y adolescentes se definen como "la preocupación, intento o acto que intencionalmente busca causarse daño a sí mismo o la muerte". Si bien la idea de

1. Departamento de Psiquiatría. Facultad de Medicina, Universidad de Concepción.

3. Residentes becarias de pediatra. Deparlamento de Pediatria, Universidad de Concepción. muerte o daño a sí mismo son parte de la definición, en el caso de los menores no es necesario un concepto maduro de la muerte'.

El suicidio es poco frecuente en menores de 10 años ${ }^{2}$, pero aumenta notoriamente en la adolescencia, llegando a ser la segunda causa de muerte en adolescentes en Estados Unidos de América?. En Chile, el mayor porcentaje de muertes en personas de 10 a 19 años $(51,7 \%)$ se debe al rubro "accidentes y violencias", donde 
Sit incluyen los suicidios ${ }^{+}$, estimándose que estos corresponden a $12 \%$ de las muertes entre 15 y 19 años

Los intentos y la ideación suicidas en la población general son aspectos bastante más difíciles de precisar. Se calcula que los intentos serian entre 30 y 100 veces más frecuentes que los suicidios logrados. Los intentos constituyen entre 10 y $30 \%$ de la carga asistencial de los servicios ambulatorios de psiquiatría infantil y su prevalencia sería de 1 a $3 \%$ en cscolares y de 3 a $15 \%$ en adolescentes ${ }^{1.3 .6-8 . ~ E n ~ u n ~ e s t u-~}$ dio nacional en adolescentes se encontró $14,2 \%$ de prevalencia de vida para los intentos suicidas?.

Pese a la creciente importancia del intento de suicidio hay pocas publicaciones en revistas nacionales sobre esta materia en diferentes poblaciones de niños y adolescentes: existen tres revisiones teóricas del tema ${ }^{2.5} 6$, un trabajo en población general ${ }^{7}$, uno en intentadores controlados en forma ambulatoria y otro en hospitalirados ${ }^{\text {te }}$.

A continuación se expone un estudio descriptivo con el que se pretende mostrar las características biodemograficas de los niños hospitalizados por intento de suicidio y las características del intento en una muestra constituida por el total de pacientes hospitalizados por intento de suicidio en el servicio de pediatría del Hospital Guillermo Grant Велavente de la ciudad de Concepción en un período de 18 meses.

\section{Material y Método}

En el periodo compreodido entre el I de octubse di2 1995 y el 31 de mayo de 1997 se registraron todos los piciertes notesador por intento de suicidio en el servicio de pediatría del Hospital Clínico Guillermo Grant Benavente de Concepción. Chile. Este servicio cuenta con horas de psiquiatria de enlace $y$ por norma notifica a la unidad de la especialidad cada vez que ingresa un menor por un jotenıo de esta naluraleza. La isfurmación contenida en e] fomnulario de interconsulta. la ficha clínica y la evaluación psicuitilurica del paciente $y$ sus padres se trascribió en un protocolo especialmente diseñado para ello en lodos los casns. Además se revisó la ficha clínica para conocer el estado actual del paciente. Con los antecedentes obtenidos se hizo un análisis descriptivo cuyos resultados se dividieron en cuatro áreas: antecedences biodemográficos de los pitcicotes; diagnósticos psiquiátricos: características del intento: estado actual del paciente y su problema clinico.

\section{Resultados}

Aspectos biodemográficos: en el período en estudio se hospitalizaron $\mathbf{3 0}$ menores por intento de suicidio, 24 eran niñas $(80 \%)$. La edad fluctuaba entre 7 años y 15 años. Tres pacientes (dos varones) tenían 10 años o menos, 12 (40\%) entre 11 y 13 años y $15(50 \%)$ adolescentes de 14 y más años. Cinco afectados $(16,7 \%)$ cursaban enseñanza media y $23(76,7 \%)$ enseñanza básica. Once víctimas $(36,7 \%)$ vivian con ambos padres, en lo que se podría considerar un hogar bien estructurado, $14(46,7 \%)$ con sólo uno de sus padres, tres $(10 \%)$ con otros familiares y dos $(6,6 \%)$ habitaban en un hogar de menores. Dieciséis (53\%) de los afectados había sufrido algún tipo de maltrato y en sólo 10 (30\%) se hizo algún diagnóstico pediátrico adjcional, siendo el más frecuente el de epilepsia que se presentó en tres casos.

Resultados de la evaluacion psiquiátrica: el diagnóstico más frecuente fue el de trastorno del estado de ánimo (depresión). Lamentablemente en tres casos no se realiz 6 evaluación psiquiátrica y en otros cuatro no se identificó un trastomo de esta indole (tabla 1).

Características del intento: el método más utilizado para intentar el suicidio fue la ingestión de fármacos, que se registró en $23(76,6 \%)$ de los intentos. Los otros dos métodos utilizados fueron la ingesta de tóxicos (raticidas, insecticidas $o$ productos de limpieza) en seis casos $(20 \%)$ y finalmente una niña intentó arrojarse a una laguna en el curso de un trastorno psicótico. Los medicamentos más usados fueron las benzodiazepinas y los neurolépticos (tabla 2).

Un alto porcentaje de los intentos tuvo lugar en la casa del menor $(n=18,60 \%)$, los demás sucedieron en el colegio $(\mathbf{n}=4)$, el hogar de menores ( $n=2$ ), otros lugares $(n=3)$ o en sitios no precisado $(n=3)$. En 19 casos $(63,3 \%)$ se trataba del primer intento y en $10(33,3 \%)$ había habido más intentos previos ( 8 sólo otro intento, uno con dos y uno con cuatro). La mayoría de los intentos fueron descritos como actos impulsivos por los afectados. Así, $17(56,6 \%)$ afirmaron no haberlo planificado con antelación, $10(30 \%)$ admitieron algunas horas de planificación y sólo en un caso describió una preparación previa de varios días. En tres casos no hubo evaluación. La mayoría (83\%) de los me- 


\section{Tabla 1}

Diagnóstico psiquiátrico en 30 niños que intentaron suicidio

\begin{tabular}{lrr}
\hline Dragnóstico & $\mathrm{n}$ & \\
\hline Trastomo del ánimo (depresión) & 16 & $5,3,3$ \\
Trastorno de personalidad & 3 & 10,0 \\
Trastomo de conduca & 2 & 6.7 \\
Otros diagnósticos* & 2 & 6,7 \\
Sin palología psiquiátrica & 4 & 13,4 \\
No evaluado & 3 & 10,0 \\
\hline Total & 30 & 100,0 \\
\hline
\end{tabular}

* trastono conversivo, psicosis orgánica

\section{Tabla 2}

Fármacos usados en 23 intentos de suicidio en niños

\begin{tabular}{lrr}
\hline Medicamentos & n & $\%$ \\
\hline Benzodiazepinas & 5 & 21,7 \\
Neurolépticos & 5 & 21,7 \\
Antidepresivos & 2 & 8,7 \\
Anticonvulsivantes & 2 & 8,7 \\
Analgésicos y relajantes musculares & 4 & 17,4 \\
Combinaciones* & 3 & 13,0 \\
Otros** & 2 & 8,7 \\
\hline Total & 23 & 100,0 \\
\hline
\end{tabular}

* Combinaciones: psicofármacos + analgésicos psicofármacos + antiinflamatorios

* Otros: esırógenos, antiasmáticos

nores reconoció algún evento precipitante del intento (tabla 3), siendo los más frecuentes los conflictos y peleas con padres, pololos y otros.

Es interesante consignar que $66,6 \%$ de los afectado tenían idea de muerte, esto es, sabían que podian morir a consecuencia del intento. Sin embargo, al estudiar la finalidad del intento, sólo la mitad se proponía dejar de vivir, mientras el resto buscaba otros objetivos (tabla 4).

Situación posterior: después del alta del hospital, 21 pacientes $(70 \%)$ asistieron a los controles programados, de los cuales 15 $(71,43 \%)$ lo hicieron con psiquiatra infantil, 3 (14,29\%) con psiquiatra infantil y psicólogo y 3 $(14,29 \%)$ sólo con psicólogo.

Al cabo de uno a 20 meses de seguimiento a contar del egreso del hospital, cuatro pacientes
(13,3\%) estaban de alta, siete $(23,3 \%)$ continuaban en control, uno había fallecido en un accidente $(3,3 \%)$, ocho $(26,6 \%)$ nunca se controlaron y se desconocía su evolución y $10(33,3 \%)$ habian abandonado el tratamiento.

\section{Comentario}

Ante la potencial mortalidad del intento de suicidio existe especial interés en determinar los factores de riesgo y los elementos ambientales precipitantes, pues así se espera diseñar estrategias preventivas bien enfocadas. El conocimiento de las características personales y familiares de los menores que intentan suicidarse y de los

\section{Tabla 3}

Eventos precipitantes en 30 intentos de suicidjo en niños

\begin{tabular}{|c|c|c|}
\hline T3̉po de precipitante & $\mathbf{n}$ & $\%$ \\
\hline Confliclo o peleas con padres & 5 & 16,6 \\
\hline Confliclos o pelea con o1ros* & 5 & 16,6 \\
\hline Conflicio o pelea coa pololo & 4 & 13,3 \\
\hline Maltrato físico & 3 & 10,0 \\
\hline Entrega de notas finales & 2 & 6.6 \\
\hline Invitación & 2 & 6.6 \\
\hline Otros desencadenantes** & 4 & 13,3 \\
\hline Sin desencadenantes & 3 & 10,0 \\
\hline No evaluado & 2 & 6.6 \\
\hline Total & 30 & 100.0 \\
\hline
\end{tabular}

\section{Tabla 4}

Finalidad de las víctimas en 30 intentos de suicidio en niños

\begin{tabular}{lrr}
\hline Finalidad & $\mathbf{n}$ & $\%$ \\
\hline Terminar con la vida & 15 & 50,0 \\
Modificar situación en la familia & 8 & 26,6 \\
Asustar a otros & 2 & 6,6 \\
Evirar situación negativa & 2 & 6,6 \\
Sin objetivo claro & 1 & 3,3 \\
No evaluado & 2 & 6,6 \\
\hline Total & 30 & 100.0 \\
\hline
\end{tabular}


sucesos previos, a través de esıudios de series clínicas como la nuestra, aporta elementos para el análisis de los mecanismos involucrados.

El perfil de los afectados, entre los que predominan las mujeres, salvo en los menores de 10 años, la edad mayor a 10 años, la desestructuración familiar, el maltrato y la ausencia de afecciones somáticas, las características del intento, los medios empleados y la finalidad coinciden con lo descrito por otros autores $1,3,5$. 6, 8-12. La alta incidencia de alteraciones psiquiátricas en los pacientes de esta serie, en especial de los trastornos del ánimo, es también coincidente con otras experiencias ${ }^{3.8}, 10.13^{-16}$ pero se contrapone con lo planteado en una revisión nacional $^{5}$

Esto permite plantear que la génesis del intento de suicidio radicaría en la interacción de factores propios del menor, con su ambiente familiar que actuaría como protector o como gatillo y con el acontecimiento de eventos vitales negativos. Sin embargo, pensamos que para interpretar adecuadamente este fenómeno debemos ser capaces de analizar cada caso en particular, ya que las interacciones específicas son muy individuales. Baste citar dos ejemplos: el de una niña de 12 años, portadora de epilepsia del lobulo temporal, que intentó autoeliminarse arrojándose a una laguna, obedeciendo a las voces alucinatorias que se lo ordenaban, $y$ el de un varón de 7 años que ingirió una sobredosis de tioridazina "para no irme de la casa de mi abuelita", ante la noticia que sería llevado donde su madre con quien nunca había vivido. Ambos ejemplos ilustran como los tres factores mencionados (individuo, familia y eventos vitales) se entrelazan para provocar el intento de suicidio.

Un aspecto de especial preocupación debería ser resolver las dificultades para brindar adecuada atención psiquiátrica a estos casos durante la hospitalización y para efectuar un seguimiento y manejo posterior apropiados. Dada la potencial letalidad del intento de suicidio, parece importante coordinar las acciones con el aporte del servicio social y los consultorios primarios a fin de niejorar las tasas de adherencia a tratamiento. La incorporación del equipo del programa salud $y$ violencia puede ser también un aporte valioso, como también la participación activa de los pediatras del servicio en el manejo de estos niños, pues ello permite mejotar aún más las posibilidades de éxito del tratamiento.

\section{Referencias}

I. Pfeffer $C$ : Suicide and suicidality. Ln. Wiener J. Texlbook of child and adolescent psychiatry. American Psychiatric Press lnc. Washington DC, USA, 1991: 507-514.

2. Ullow F: Prevención del suicidio en niños y adolescentes. Rev Chil Pediatr 1994; 65: I78-183.

3. Brens $D$, Kolko $D$ : Suicide and suicidal behavior in children and adolescents, ln: Gorlinkel B, Carlson $G$, Weller P: Psychiatric disorders in children and adolescents. W.B. Saunders Company, Philadelphia, USA, 1990: 372-391.

4. Kaentpfer AM: Adolescencia y Salud Publica. Aspectos Sociodemográficos. In: Florenzano R. Maddaleno M, Bobadilla E. (Eds). La salud del Adolescente en Chile. Editorial CPU, Santiago. 1988: $17-40$.

5. Ulloa $F$ : Tentativa y consumación de suicidio en nifos y udolescentes. Rev Chil Pediatr 1993; 64: 272 276.

6. De la Barra $F$ : Conducla suicida en niños y adolescentes. Pediatría al día. (Santiago) 1989; 5: 152-158.

7. Sotvo L: Ideación suicida e intento de suicidio en adolescentes de enseñanza media de la Comuna de Concepción (Tesis para Optar a la Especialidad de Psiquiatría). Facultad de Medicina, Universidad de Concepción 1995.

8. Ferkusson $D$, Lynskey $M$ : Suicide atempts and suicidal idealion in a birth cohort of 16 year old New Zealanders. J Am Acad Child Adolesc Psychiatry $1995 ; 34: 1308-1317$.

9. Vatdivia $M$, Arayo $F M$ : Intento de suicidio en nin̆os: atención en Policlínico de Psiquiatría Infantil (Presenlado en el XXXVil Congreso Chileno de Pediatria). Rev Chil Pediatr 1996; 67 (Número especial): 56.

10. Rona E. Wentin $L$. Tentalivas de suicidio en niños y adolescentes. Rev Chil Pediatr. 1986; 57: 227-230.

11. Altamirano. P: Comportamiento suicida en la atolescencia. ¿Causas y explicaciones? Pediatría al Día 1995; 11: $31-33$.

12. Pfeffer C: Suicidal behavior in children and adolescent: causes and mangement: In: Lewis $M$, (Ed) Child and Adolescent Psychriatry a compretensive Textbook. Williams and Wilkins, Baltimore, USA, 1996: 666673

13. Fergusson $D$, Lynskey $M$ : Childhood circunstances adolescent adjustment and suicide attempis in a New Zealand birth cohort. J Am Acad Child Adalesc Psychiatry $1995 ; 34: 612-622$

14. Beautrans A. Joyce P: Prevalence and comorbidity of mental disorders in persons making serious suicide attempts: A case control study. Am J Psychiatry 1996; 153: $1009-1014$

15. Wend E. Lin I: The Associations of Suicide Attentpts in Adolescents Pediatrics 1997; $99: 791796$.

16. Roberts $R$, Chen $Y$ : Depressive symptoms and suicidal ideation among mexican-origin and anglo adolescents. J Am Acad Child Adolesc Psychiatry 1995: 34: 81-90. 